\title{
Mobile Learning Technologies
}

\author{
Khalil Alsaadat \\ Department of Educational Policies, College of Education, King Saud University, Riyadh, Saudi Arabia
}

\begin{tabular}{l}
\hline \hline Article Info \\
\hline Article history: \\
Received Aug 9, 2017 \\
Revised Feb 20, 2018 \\
Accepted Apr 21, 2018 \\
\hline Keyword: \\
Mobile learning \\
Technology
\end{tabular}

\begin{abstract}
Technological development have altered the way we communicate, learn, think, share, and spread information. Mobile technologies are those that make use of wireless technologies to gain some sort of data. As mobile connectedness continues to spread across the world, the value of employing mobile technologies in the arena of learning and teaching seems to be both self-evident and unavoidable The fast deployment of mobile devices and wireless networks in university campuses makes higher education a good environment to integrate learners-centered m-learning . this paper discusses mobile learning technologies that are being used for educational purposes and the effect they have on teaching and learning methods.
\end{abstract}

\section{Corresponding Author:}

Khalil Alsaadat, Department of Educational Policies, College of Education, King Saud University, Riyadh, Saudi Arabia Email: alsaadat@gmail.com

\section{INTRODUCTION}

Because m-Learning is such a new field the research is still in a stage where different categories of m-Learning pedagogy are being developed, identified, and researched. With this developmental stage in mind, the very existence of m-Learning, not to mention its growing application, is directly tied to the growth of mobile technology. This fact is why it is so important for researchers and practitioners to be familiar with mobile technology applicable to m-Learning. It simply is not possible for someone to log onto a learning management system (LMS) wirelessly from a personal digital assistant (PDA) if wireless networks don't exist or if PDAs do not support wireless connectivity [1].

\section{THE MEANING OF MOBILE LEARNING}

The term mobile learning (m-learning) refers to the use of mobile and handheld IT devices, such as Personal Digital Assistants (PDAs), mobile telephones, laptops and tablet PC technologies, in teaching and learning. As computers and the internet become essential educational tools, the technologies become more portable, affordable, effective and easy to use. This provides many opportunities for widening participation and access to ICT, and in particular the internet. Mobile devices such as phones and PDAs are much more reasonably priced than desktop computers, and therefore represent a less expensive method of accessing the internet (though the cost of connection can be higher). The introduction of tablet PCs now allows mobile internet access with equal, if not more, functionality than desktop computers [2].

M-Learning, or "mobile learning", has different meanings for different communities. Although related to e-learning and distance education, it is distinct in its focus on learning across contexts and learning with mobile devices. One definition of mobile learning is: Learning that happens across locations, or that 
takes advantage of learning opportunities offered by portable technologies. In other words, mobile learning decreases limitation of learning location with the mobility of general portable devices.

The term covers: learning with portable technologies, where the focus is on the technology (which could be in a fixed location, such as a classroom); learning across contexts, where the focus is on the mobility of the learner, interacting with portable or fixed technology; and learning in a mobile society, with a focus on how society and its institutions can accommodate and support the learning of an increasingly mobile population that is not satisfied with existing learning methodologies.

M-learning is convenient, in the sense that it is accessible virtually from anywhere(class, taxi, laundry room, bathroom) which provides access to all the different learning materials available. Moreover, it is collaborative; that is sharing is almost instantly among everyone using the same content, which will in turn also lead to receiving instant feedback and tips. M-Learning also brings strong portability by replacing books and notes with small RAMs, filled with tailored learning contents. In addition, this kind of learning is engaging and fun. With this kind of learning, it is much easier to combine gaming and learning for a more effective and entertaining experience [3].

Access : How widely available is the wireless network that will distribute the mobile content?

Richness : Do pages load quickly? Do animations play in a smooth and seamless manner? Does the streaming media (media that is consumed-read, heard, viewed-while it is being delivered) flow at a sufficiently rapid rate?

Efficiency : How large is the client that will be required to make use of a particular media player? How fast will the application load and play?

Flexibility : Will the application be viewable on a variety of devices? Can content designed for use with one kind of device or operating system be played on other devices with some expectation of comparable quality?

Security : Is the interactive mobile device protected from worms and viruses? Is the shared content protected from being intercepted by unintended recipients?

Reliability : Will content be displayed in a consistent manner, regardless of the browser, device, and screen size?

Interactivity : Does the application allow users to interact freely with the display and the content[4]. Through the use of mobile learning, user can access learning content without any borders so that it can be accessed at any time so that it can be accessed at any time with interesting illustration[5].

\section{MOBILE HARDWARE}

Various types of derivative information have been increasing exponentially, based on mobile devices and social networking sites, and the information technologies utilizing them have also been developing rapidly. Technologies to classify and analyze such information are as important as data generation [6]. Caudill said the first device that comes to mind when mobile hardware is discussed is the PDA. These devices offer many of the features of a full-size laptop computer but in a package that fits in a pocket. As discussed, mobility is a primary component of m-Learning hardware, and few devices offer the combination of mobility and features that the PDA does.

From the start, the PDA experience lends itself to being ideal for the m-Learning environment. Whether a Palm or PocketPC operating system, a PDA will start up almost instantly, as opposed to the boot process that is required for a larger computer. This advantage by itself is a significant one; if a learner wants to check e-mail or reply to a message board while in between appointments, the time spent booting up and shutting down a traditional computer platform is a very real deterrent, the PDA interface eliminates that wasted time.

Once the PDA is active, it provides a wide variety of applications that in the past were not available on mobile devices. Andronico and colleagues 2003, investigate three areas of applications using PDAs in the m-Learning environment:

The use of PDA as an enhanced organizer, by uploading/ downloading data with the central system in order to align periodically or on demand the agenda of the user (teacher, student, or other actors of the system) with all the academic appointments. This will imply the integration of the data schema of the agenda software of the portable device with the data coming from the central system. The browsing of newsgroups managed by the central Learning management system (LMS) on the PDA screen, in case the user has no keyboard attached to the portable device, or the full interaction with the newsgroup in the other case. 
The browsing of the LMS web pages where it is possible to download the educational material and consult it with specific viewers (at the moment those related with the Office suite and with Acrobat PDF format [1].

a. First, producers of hardware and operating systems often minimise costs and maximise effect through product development partnerships

b. Second, demand from consumers and businesses is the influence on the type of product being developed, with the common requests being: easier to use, smaller, faster, smarter, and greater security. This has resulted in advancements such as multiple security layers, Bluetooth, car and business kits, hands free and infrared/ data cables. When asked about the future applications or capabilities that are planned for existing products, the response was: 'smaller, faster, better, cheaper, and more wireless technology to send bigger files faster.'

What percentage of mobile technologies are purchased for business purposes and what percentage is purchased for consumer use? The following quote tells the story:

There is such a cross-over between personal and business use we're unable to tell them apart. Higher end products (i.e., Bluetooth or wireless, products with extra security, or products containing enterprise solutions) are marketed only to business clients, but the simplest phone can be used for business as well as personal purposes

Much is made of the potential of m-Learning, but what is actually happening from a developer perspective? The two following quotes illustrate the developers' insights:

a. A flash-based mobile interface is now being produced for m-Learning, so that animated material can be used on mobile phones; the technology is now moving quickly to respond to the increasing speed of mLearning uptake

b. As an add-on to other modes of deliver, m-Learning will increase. But it won't replace other forms of eeducation because screens are too small and hard to read, and if you make them bigger, the device isn't as mobile. m-Learning is most useful when it's in a mobile, field environment[7].

Traxler turned our attention to some categories of mobile learning that are emerging,he listed the following:

a. Technology-driven mobile learning - Some specific technological innovation is deployed in an academic setting to demonstrate technical feasibility and pedagogic possibility

b. Miniature but portable e-Learning - Mobile, wireless, and handheld technologies are used to re-enact approaches and solutions already used in 'conventional' e-Learning, perhaps porting some e-Learning technology such as a Virtual Learning Environment (VLE) to these technologies or perhaps merely using mobile technologies as flexible replacements for static desktop technologies

c. Connected classroom learning - The same technologies are used in classroom settings to support collaborative learning, perhaps connected to other classroom technologies such as interactive whiteboards

d. Informal, personalised, situated mobile learning - The same technologies are enhanced with additional functionality, for example location-awareness or video-capture, and deployed to deliver educational experiences that would otherwise be difficult or impossible

e. Mobile training/ performance support - The technologies are used to improve the productivity and efficiency of mobile workers by delivering information and support just-in-time and in context for their immediate priorities (for an early account, see Gayeski, 2002)

f. Remote/ rural/ development mobile learning - The technologies are used to address environmental and infrastructural challenges to delivering and supporting education where 'conventional' e-Learning technologies would fail, often troubling accepted developmental or evolutionary paradigms

Mobile distance learning could fall into any of these categories (with the exception of the 'connected classroom learning'); how it develops will depend in part on the affordances of any given situation. These affordances might include:

a. Infrastructure, meaning power supply, postal services, Internet connectivity, etc.

b. Sparsity, giving rise to infrequent face-to-face contact, lack of technical support, etc.

c. The wider policy agenda including lifelong learning, inclusion (of rural areas for example), assistivity, participation, and access

d. Mobile distance learning within a framework of blended distance learning and the affordances of other delivery and support mechanism [8].

\section{MOBILE NETWORKING}

Caudill noticed that the most prevalent and most widely recognized mobile networking technology is the IEEE 802.11 specification, commonly called Wi-Fi. Wi-Fi works by using a series of access points, which are transmitter/ receiver stations that wireless devices can connect to via their own Wi-Fi networking 
card. Initially seen as external cards that were used in a Personal Computer Memory Card International Association (PCMCIA) slot on laptops, Wi-Fi networking devices are now being integrated into standardsize PDAs and even smaller platforms such as mobile gaming devices. If a mobile device does not have a built-in Wi-Fi card there are a wide variety of add-on cards available, some small enough to fit into the SD slots on handheld devices. Thus, many devices not originally configured to access wireless networks can be converted to do so.he aso said: at 11 Megabits per second (Mbps), and 802.11g (g), which transmits at 54 Mbps. Both of these common standards are interoperable, There are two widely used standards, 802.11b (b), which transmits meaning that a g device can operate at a slower speed on a b network, and a b device can access a g network. Most devices today that include built-in Wi-Fi connectivity are using either the g or b standard, as are most publicly accessible Wi-Fi access points, which are referred to as hotspots[1].

\section{DISCUSSION}

The distance learning is an instruction system which has various and independent instruction environment, customized lecture design, method and techniques. Distance learning has been improving from letter learning to mobile learning [9]. Because of its high mobility and spontaneity, m-learning offers learners access to learning objects and resources that are distributed around us. In the 'anytime, anywhere, learning area. what really matters is whether learners can access the right resources at the right time in the right place. Learners should be able to interact with the learning objects both in the real world and in the virtual world. Apart from that, the ultimate goal of m-learning is to enable mobile devices to offer individualized guidance and support during the learning process, and replace the one-size-fits-all receptive style of learning. It enables students to walk out of the classroom and actively explore their learning environment to gain more experience in collaboration and problem solving [10].

Traxler argued that mobile education, however innovative, technically feasible, and pedagogically sound, may have no chance of sustained, wide-scale institutional deployment in higher education in the foreseeable future, at a distance or on site. This is because of the strategic factors at work within educational institutions and providers. These strategic factors are different from those of technology and pedagogy. They are the context and the environment for the technical and the pedagogic aspects. They include resources (that is, finance and money but also human resources, physical estates, institutional reputation, intellectual property, and expertise) and culture (that is, institutions as social organisations, their practices, values and procedures, but also the expectations and standards of their staff, students and their wider communities, including employers and professional bodies).

Implementing wireless and mobile education within higher education must address these social, cultural, and organisational factors. They can be formal and explicit, or informal and tacit, and can vary enormously across and within institutions. Within institutions, different disciplines have their own specific cultures and concerns, often strongly influenced by professional practice in the 'outside world' - especially in the case of part-time provision and distance learning. Because most work in mobile learning is still in the pilot and/ or trial phase, any explorations of wider institutional issues are still tentative [11].

Peters in alsaadat asked Is the promise of mobile technologies as a trigger to generate learning cultures realistic? And is m-Learning any more likely to increase interest in learning than any other form of delivery? Articles about the link between mobile technologies and learning organisations appear to fall into three categories:

1. A database focus that captures organisational knowledge

2. A human systems focus that allows synchronous communication and information sharing at the worksite

3. A learning development focus that suggests that learning about new technologies generates a more general drive for learning

The database focus has, to a large degree, become the accepted wisdom in organisations that use structured processes to collect, codify, and manage knowledge. Mobile technologies have the potential to collect a greater range and percentage of data, through recording of activity on the device (and subsequent analysis of the patterns of access to specific information or information sources) and through the reduction of paper-based records as electronic systems replace paper in the field.

Peters thinks that the capacity of mobile technology to deliver synchronous communication and knowledge-sharing can provide benefits to human (or soft) systems. Evidence of these benefits has been reported by Ragus 2004, who found that m-Learning encouraged simultaneous personal development, such as networking and socialisation, outside of normal working groups - an unexpected, and positive result of the m-Learning trials [12]. 


\section{CONCLUSION}

Mobile technology, both hardware and networking applications, is a necessary component for the existence of m-Learning. As instructors and designers, practitioners of m-Learning need to be fluent in the use of these technologies and cognizant of what technologies their learner population has access to. Application of specific pedagogical theories is directly connected to the technologies in use in a m-Learning system and as such, design of m-Learning environments demands a systems approach, where development accounts for all aspects of the environment. As technology continues to improve and innovate the options open to m-Learning will expand; the key is to focus on the fact that the goal of m-Learning is to facilitate learning, no matter what form the delivery may take [1].

\section{REFERENCES}

[1] Caudil,Jason g,2007."The growth of m learning and the growth of mobile computing":paralle development"

[2] Exellence gateway.org.uk

[3] Wikipedia (reference:http://www.grayharriman.com/mlearning.htm)

[4] Wagner ellen D.2005,"enabling mobile learning" ,Educause review,V40,N3,pp40-53.

[5] Setiawan, alexsander. hanreas handojo.hadi,renadra, "indonisian culture learning application based on android",2017, international journal of electrical and computer engineering, vol 7, No 1, 2017

[6] Jung se hoon.chan kim,jong.bo sim chun,"prediction data processing scheme using on artificial network and data clustering for big data", international journal of electrical and computer engineering, Vol 6,No 1,2016.

[7] Peters,2007."M learning:positioning educators for a mobile,connected future", the international review of research in open and distance learning,VOL8,NO2.

[8] Traxler,2007,"Defining,Discussing and Evaluating mobile learning : the moving finger writes" ,the international review of research in open and distance learning. VOL8,NO2.

[9] Soykan,emran,"the review of puplished articles on mobile learning area in Ebesco data base",procedia social and behavioral science, $\mathrm{Vol} 182,2015$

[10] Shin,ju ling . chun chu ,hui,hawang ,gwo gen.kinshuk,"an investigation of attitudes of students and teachers about participating in a context aware ubiquitous learning activity",British journal of educational technology, Vol 42 ,No 3,2011.

[11] Alsaadat,khalil, "m learning and college education",European journal of education studies, Vol 3, Issue 5 ,2017.

[12] Alsaadat,khalil,"the importance of m learning in the educational arena",European journal of open education and $e$ learning studies, Vol 2, Issue $1,2017$. 